\begin{tabular}{|c|l|}
\hline Title & On characterizations of the image of Gelfand transform of commutative Banach algebras \\
\hline Author(s) & Inoue, J.; Takahashi, S.-E \\
\hline Citation & Hokkaido University Preprint Series in Mathematics, 374, 1-30 \\
\hline Issue Date & 1997-4-1 \\
\hline DOI & 10.14943/83520 \\
\hline Doc URL & http://hdl.handle.net/2115/69124 \\
\hline Type & bulletin (article) \\
\hline File Information & pre374.pdf \\
\hline
\end{tabular}

Instructions for use 


\section{On characterizations of the image of Gelfand transform of commutative Banach algebras}

J. Inoue and S.-E. Takahashi

Series \#374. April 1997 


\section{HOKKAIDO UNIVERSITY PREPRINT SERIES IN MATHEMATICS}

\#350 F. Hiroshima, Weak coupling limit with a removal of an ultraviolet cut-off for a Hamiltonian of particles interacting with a massive scalar field, 21 pages. 1996.

\#351 T. Nakazi and Y. Watatani, Invariant subspace theorems for subdiagonal algebras, 22 pages. 1996.

\#352 Y. Nishiura and H. Suzuki, Nonexistence of stable turing patterns with smooth limiting interfacial configurations in higher dimensional spaces, 21 pages. 1996.

\#353 Y.-G. Chen, Y. Giga and K. Sato, On instant extinction for very fast diffusion equations, 9 pages. 1996.

\#354 A. Gyoja and H. Yamashita, Associated variety, Kostant-Sekiguchi correspondence, and locally free $U(\mathrm{n})$-action on Harish-Chandra modules, 25 pages. 1996.

\#355 G. Ishikawa, Topology of plane trigonometric curves and the strangeness of plane curves derived from real pseudo-line arrangements, 18 pages. 1996.

\#356. N.H. Bingham and A. Inoue, The Drasin-Shea-Jordan theorem for Hankel transforms of arbitrarily large order, 13 pages. 1996.

\#357 S. Izumiya, Singularities of solutions for first order partial differential equations, 20 pages. 1996.

\#358 N. Hayashi, P.I. Naumkin and T. Ozawa, Scattering theory for the Hartree equation, 14 pages. 1996.

\#359 I. Tsuda and K. Tadaki, A logic-based dynamical theory for a genesis of biological threshold, 49 pages. 1996.

\#360 I. Tsuda and A. Yamaguchi, Singular-continuous nowhere-differentiable attractors in neural systems, 40 pages. 1996.

\#361 M. Nakamura and T. Ozawa, Low energy scattering for nonlinear Schrödinger equations in fractional order Sobolev spaces, 17 pages. 1996.

\#362 I. Nakamura, Hilbert schemes and simple singularities $E_{6}, E_{7}$ and $E_{8}, 21$ pages. 1996.

\#363 T. Mikami, Equivalent conditions on the central limit theorem for a sequence of probability measures on $R, 7$ pages. 1996 .

\#364 S. Izumiya and T. Sano, Generic affine differential geometry of space curves, 23 pages. 1996.

\#365 T. Tsukada, Stability of reticular optical caustics, 12 pages. 1996.

\#366 A. Arai and M. Hirokawa, On the existence and uniqueness of ground states of a generalized spin-boson model, 40 pages. 1996.

\#367 A. Arai, A class of representations of the *algebra of the canonical commutation relations over a Hilbert space and instability of embedded eigenvalues in quantum field models, 12 pages. 1996.

\#368 K. Ito, BV-solutions of a hyperbolic-elliptic system for a radiating gas, 33 pages. 1997.

\#369 M. Nakamura and T. Ozawa, Nonlinear Schrödinger equations in the Sobolev space of critical order, 20 pages. 1997.

\#370 N.H. Bingham and A. Inoue, An Abel-Tauber theorem for Hankel transforms, 8 pages. 1997.

\#371 T. Nakazi and H. Sawada, The commutator ideal in Toeplitz algebras for uniform algebras and the analytic structure, 9 pages. 1997.

\#372 M.-H. Giga and Y. Giga, Stability for evolving graphs by nonlocal weighted curvature, 70 pages. 1997.

\#373 T. Nakazi, Brown-Halmos type theorems of weighted Toeplitz operators, 14 pages. 1997. 


\title{
On characterizations of the image of Gelfand transform of commutative Banach algebras
}

\author{
Jyunji Inoue* \\ Department of Mathematics, Faculty of Science \\ Hokkaido University, Sapporo 060, Japan \\ and
}

Sin-Ei Takahasi

Department of Basic Technology Applied Mathematics and Physics

Yamagata University, Yonezawa 992 Japan

Dedicated to Professor Satoru Igari on his 60th birthday

\begin{abstract}
In this paper, we propose a new method to characterize in terms of "quasi - topology" the image of Gelfand transform of commutative Banach algebras, which were inspired by the works of R. Doss in 1976-77. Generalizations of Bochner-Schoenberg-Eberlein theorem to general commutative Banach algebras (which play very important roles in this paper) are also considered. Our method is applied in this paper to some concrete Banach algebras such as, group algebras $L^{1}(G)$, ideals and quotient algebras of $L^{1}(G)$, Segal algebras $L^{1}(G) \cap L^{p}(G)(1<p<\infty)$ for locally compact abelian groups $G$ and $C^{*}$-algebra $C_{0}(\Omega)$ on locally compact Hausdorff spaces $\Omega$ etc.

${ }^{*}$ E-mail: jinoue@math.hokudai.ac.jp

${ }^{\circ}$ The authors are partly supported by the Grants-in-Aid for Scientific Research, The Ministry of Education, Science and Culture, Japan.
\end{abstract}




\section{Introduction and Terminology}

Throughout this paper, $A$ denotes a commutative semisimple Banach algebra with Gelfand space $\Phi_{A}$. In $\Phi_{A}$, we consider the Gelfand topology, which makes $\Phi_{A}$ a locally compact Hausdorff space. $\Phi_{A}$ is compact if and only if $A$ has a unit element. $G$ denotes a locally compact abelian group (LCA-group) with the dual group $\hat{G}$.

This paper is inspired by the following two theorems of R. Doss $[2,3]$.

Theorem A ([2]). A continuous function $\sigma$ on $\hat{G}$ is the Fourier-Stieltjes transform of an absolutely continuous measure if and only if, whatever be $\varepsilon>0$, there is a compact set $K$ and $a \delta>0$ such that, for any trigonometric polynomial $p(x)=\sum c_{i}\left(-x, \gamma_{i}\right)$, the relations

$$
\|p\|_{\infty} \leq 1, \int_{K}|p(x)| d x<\delta \text { imply }\left|\sum c_{i} \sigma\left(\gamma_{i}\right)\right|<\varepsilon .
$$

Theorem B ([3]). A continuous function $\sigma$ on $\hat{G}$ is the Fourier-Stieltjes transform of an absolutely continuous measure if and only if

(i) there is a constant $M$ such that for every polynomial

$$
p(x)=\sum c_{i}\left(-x, \gamma_{i}\right), \quad \gamma_{i} \in \hat{G},
$$

the relation $\|p\|_{\infty} \leq 1$ implies $\left|\sum c_{i} \sigma\left(\gamma_{i}\right)\right| \leq M$;

(ii) whatever be $\varepsilon>0$ there is a compact set $K$ in $\hat{G}$ such that, for every polynomial $p(x)=\sum c_{i}\left(-x, \gamma_{i}\right), \gamma_{i} \in \hat{G} \backslash K$, the relation $\|p\|_{\infty} \leq 1$ implies $\left|\sum c_{i} \sigma\left(\gamma_{i}\right)\right| \leq \varepsilon$.

In the above theorems, we can regard $\sigma$ as a linear functional on the space of trigonometric polynomials, and interpret the conditions on $\sigma$ as a certain continuity of $\sigma$ on the space of trigonometric polynomials. From this point of view, we will consider the characterizations of the image of Gelfand transform of a commutative Banach algebra.

$\operatorname{span}\left(\Phi_{A}\right)$ expresses the linear span of $\Phi_{A}$ in the dual space $A^{*}$ of $A$. Of course, arbitrary element $p$ of $\operatorname{span}\left(\Phi_{A}\right)$ has the unique expression $p=\dot{\Sigma}_{\varphi} \hat{p}(\varphi) \varphi$, where $\hat{p}$ is a complex-valued function on $\Phi_{A}$ with finite support. 
Definition 1.1. A family $\mathcal{Q}$ consisting of subsets of $\operatorname{span}\left(\Phi_{A}\right)$ is called a quasitopology for $\operatorname{span}\left(\Phi_{A}\right)$ provided the following two conditions are satisfied:

(i) Every $U \in \mathcal{Q}$ contains the origin (zero-functional) of $\operatorname{span}\left(\Phi_{A}\right)$.

(ii) For every $U, V \in \mathcal{Q}$, there exists a $W \in \mathcal{Q}$ such that $W \subset U \cap V$.

In this case, each member of $\mathcal{Q}$ is called a quasi-neighbourhood for $\operatorname{span}\left(\Phi_{A}\right)$. Obviously, $\mathcal{Q}$ naturally forms a net by the set inclusion relations.

Definition 1.2. Let $\mathcal{P}$ and $\mathcal{Q}$ be two quasi-topologies for $\operatorname{span}\left(\Phi_{A}\right)$. If, for each $U \in \mathcal{P}$ there exists a $V \in \mathcal{Q}$ such that $V \subset U$, then $\mathcal{Q}$ is said to be stronger than $\mathcal{P}$ and denote this relation by $\mathcal{P} \leq \mathcal{Q}$ or $\mathcal{Q} \geq \mathcal{P}$. If $P \leq \mathcal{Q}$ and $\mathcal{Q} \leq \mathcal{P}$, then $\mathcal{P}$ is said to be equivalent to $\mathcal{Q}$, and denote this by $\mathcal{P} \cong \mathcal{Q}$. If $\mathcal{P} \leq \mathcal{Q}$ and $\mathcal{P}$ is not equivalent to $\mathcal{Q}$,

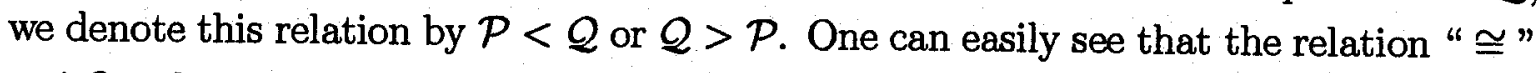
satisfies the equivalent conditions, and the relation " $\leq$ " is reflexive and transitive.

Definition 1.3. Suppose that $\mathcal{Q}$ is a quasi-topology for $\operatorname{span}\left(\Phi_{A}\right)$. A continuous complex-valued function $\sigma$ on $\Phi_{A}$ is said to induce a $\mathcal{Q}$-continuous linear functional on $\operatorname{span}\left(\Phi_{A}\right)$ provided for each $\varepsilon>0$, there exists a $U \in \mathcal{Q}$ such that

$$
\left|\sum_{\varphi \in \Phi_{A}} \hat{p}(\varphi) \sigma(\varphi)\right|<\varepsilon \quad(p \in U)
$$

$C\left(\Phi_{A} ; \mathcal{Q}\right)$ denotes the set of all continuous functions on $\Phi_{A}$ which induces $\mathcal{Q}$ continuous linear functional on $\operatorname{span}\left(\Phi_{A}\right)$. For a quasi-topology $\mathcal{Q}$ for $\operatorname{span}\left(\Phi_{A}\right)$, one can easily see that $C\left(\Phi_{A} ; \mathcal{Q}\right)$ becomes a linear space in the natural way, and that $P \leq \mathcal{Q}$ implies $C\left(\Phi_{A} ; \mathcal{P}\right) \subseteq C\left(\Phi_{A} ; \mathcal{Q}\right)$. But the converse does not necessarily hold.

Now denote by $\hat{x}$ the Gelfand transform of $x \in A$, and let $\hat{A}=\{\hat{x}: x \in A\}$. Also, let us denote by $\mathcal{Q}_{0}=\mathcal{Q}_{0}(A)$ a quasi-topology for $\operatorname{span}\left(\Phi_{A}\right)$ determined by quasineighbourhoods of the form

$$
U\left(x_{1}, \ldots, x_{n} ; \varepsilon\right)=\left\{p \in \operatorname{span}\left(\Phi_{A}\right):\left|p\left(x_{i}\right)\right|<\varepsilon, i=1, \ldots, n\right\},
$$

where $\left\{x_{1}, \ldots, x_{n}\right\}$ is any finite set of elements from $A$ and $\varepsilon>0$. Of course, $\mathcal{Q}_{0}$ is a local base of the origin for the relative weak*-topology for $\operatorname{span}\left(\Phi_{A}\right)$. We shall see later that $\mathcal{Q}_{0}$ is the smallest quasi-topology among the quasi-topologies $\mathcal{Q}$ for $\operatorname{span}\left(\Phi_{A}\right)$ satisfying $\hat{A}=C\left(\Phi_{A} ; \mathcal{Q}\right)$. In the case of $A=L^{1}(G)$, the group algebra of $G$, we can identify $\Phi_{A}$ with $\hat{G}$, and also identify $\operatorname{span}\left(\Phi_{A}\right)$ with $\operatorname{span}(\hat{G})$, the set of all trigonometric 
polynomials on $G$. For $f \in L^{1}(G)$, the Fourier transform $\hat{f}$ is equals to the Gelfand transform of $f$, and $L^{1}(G)^{\wedge}=\left\{\hat{f}: f \in L^{1}(G)\right\}$.

We denote by $\mathcal{Q}_{D}=\mathcal{Q}_{D}\left(L^{1}(G)\right)$ a family consists of the sets of the form

$$
U_{K, \varepsilon}=\left\{p \in \operatorname{span}(\hat{G}):\|p\|_{\infty} \leq 1, \int_{K}|p(x)| d x<\varepsilon\right\},
$$

where $K$ is any compact subset of $G$ and $\varepsilon>0$. In this case, it is easy to see that $\mathcal{Q}_{D}$ satisfies the conditions (i) and (ii) of Definition 1.1, but does not satisfy the following condition:

(iii) For each $U \in \mathcal{Q}_{D}$, there exists a $V \in \mathcal{Q}_{D}$ such that for any $p \in V$ there exists a $W_{p} \in \mathcal{Q}_{D}$ with $p+W_{p} \subset U$.

Therefore, $\mathcal{Q}_{D}$ is a quasi-topology for $\operatorname{span}\left(\Phi_{A}\right)$, but it is not a local base of the origin for any topology compatible with the operations on $\operatorname{span}\left(\Phi_{A}\right)$.

Observe that Theorem A asserts that $L^{1}(G)^{\wedge}=C\left(\hat{G} ; \mathcal{Q}_{D}\right)$, and by Lemma 2.1 of the next section, we have $L^{1}(G)^{\wedge}=C\left(\hat{G} ; \mathcal{Q}_{0}\right)$. However, as observed later, we have $\mathcal{Q}_{0}<\mathcal{Q}_{D}$ whenever $G$ is infinite, and we could say that $L^{1}(G)^{\wedge}=C\left(\Phi_{A} ; \mathcal{Q}_{D}\right)$ describes $L^{1}(G)^{\wedge}$ more precisely than $L^{1}(G)^{\wedge}=C\left(\hat{G} ; \mathcal{Q}_{0}\right)$. From this point of view, our main interest in this paper lies in, given a concrete Banach algebra $A$, to discover and study a quasi-topology $\mathcal{Q}$ for $\operatorname{span}\left(\Phi_{A}\right)$ which satisfies $\mathcal{Q}_{0}<\mathcal{Q}$ and $\hat{A}=C\left(\Phi_{A} ; \mathcal{Q}\right)$.

In $\S 2$, we prove basic lemmas which are fundamental in considering quasi-topologies in this paper. In $\S 3$, brief introduction on induced algebras and $B E S$-algebras are given. An analogy of Theorem A for algebras $A=C_{o}(\Omega)$ in the context of quasi-topologies is pursued in $\S 4$. In $\S 5$, another quasi-topology $\mathcal{Q}_{c}$ for $\operatorname{span}\left(\Phi_{A}\right)$ is introduced, whose definition for general commutaive Banach algebras $A$ was suggested by Theorem $\mathrm{B}$.

The remainder sections of the paper are devoted to the study of the properties of Banach algebras with respect to this quasi-topology $\mathcal{Q}_{c}$. In $\S 6$, conditions on $A$ to assure $\hat{A}=C\left(\Phi_{A} ; \mathcal{Q}_{c}\right)$ are pursued. Some concrete Banach algebras are studied in the context of the quasi-topology $\mathcal{Q}_{c}$ in $\S 7$ and $\S 8$; in $\S 7$, ones which satisfy $\hat{A}=C\left(\Phi_{A} ; \mathcal{Q}_{c}\right)$, and in $\S 8$, ones which satisfy $\hat{A} \neq C\left(\Phi_{A} ; \mathcal{Q}_{c}\right)$. 


\section{Basic Lemmas}

The following lemmas are basic in considering the quasi-topologies in this paper.

Lemma 2.1. (i) $\hat{A}=C\left(\Phi_{A} ; \mathcal{Q}_{0}\right)$. (ii) If $\mathcal{Q}$ is a quasi-topology for $\operatorname{span}\left(\Phi_{A}\right)$ such that $\hat{A} \subseteq C\left(\Phi_{A} ; \mathcal{Q}\right)$ then $\mathcal{Q}_{0} \leq \mathcal{Q}$.

Proof. (i) For each $x \in A$, set $\tilde{x}(p)=p(x)\left(p \in \operatorname{span}\left(\Phi_{A}\right)\right)$. Then $\tilde{A}=\{\tilde{x}: x \in A\}$ is a separating linear subspace of $\left(\operatorname{span}\left(\Phi_{A}\right)\right)^{\prime}$, the linear space consisting of linear functionals on $\operatorname{span}\left(\Phi_{A}\right)$, and hence the $\tilde{A}$-topology makes $\operatorname{span}\left(\Phi_{A}\right)$ into a locally convex linear topological space whose dual space is $\tilde{A}$. (cf. Rudin [15] p.64, 3.10 Theorem).

Let $\sigma$ be a continuous function on $\Phi_{A}$, and set

$$
\tilde{\sigma}(p)=\sum_{\varphi} \hat{p}(\varphi) \sigma(\varphi) \quad\left(p \in \operatorname{span}\left(\Phi_{A}\right)\right)
$$

Then $\tilde{\sigma}$ is a linear functional on $\operatorname{span}\left(\Phi_{A}\right)$. If $\sigma \in C\left(\Phi_{A}, \mathcal{Q}_{0}\right)$, then $\tilde{\sigma}$ is continuous for the $\tilde{A}$-topology, and from above we can find an element $x \in A$ such that $\tilde{\sigma}=\tilde{x}$, so $\sigma=\hat{x}$. Conversely, we can easily see that every $\hat{x}(x \in A)$ is $\mathcal{Q}_{0}$-continuous on $\operatorname{span}\left(\Phi_{A}\right)$.

(ii) Let $\mathcal{Q}$ be a quasi-topology for $\operatorname{span}\left(\Phi_{A}\right)$ such that $\hat{A} \subseteq C\left(\Phi_{A} ; \mathcal{Q}\right)$. Suppose that $\mathcal{Q}_{0} \not \mathbb{Q}$. Then there exists a $U\left(x_{1}, \ldots, x_{n} ; \varepsilon_{0}\right) \in \mathcal{Q}_{0}$ such that

$$
V \not \subset U\left(x_{1}, \ldots, x_{n} ; \varepsilon_{0}\right)
$$

for every $V \in \mathcal{Q}$. Since each $\hat{x}_{i}$ is $\mathcal{Q}$-continuous by hypothesis, we can find a quasineighbourhood $V_{i} \in \mathcal{Q}$ such that $\left|p\left(x_{i}\right)\right|<\varepsilon_{0}$ for all $p \in V_{i}$. Then take a quasineighbourhood $V_{0} \in \mathcal{Q}$ such that $V_{0} \subset V_{1} \cap \ldots \cap V_{n}$. By (1), we can find an element $p_{0} \in V_{0} \backslash U\left(x_{1}, \ldots, x_{n} ; \varepsilon_{0}\right)$, and hence $\left|p_{0}\left(x_{i_{0}}\right)\right| \geq \varepsilon_{0}$ for some $i_{0}\left(1 \leq i_{0} \leq n\right)$. But since $p_{0} \in V_{0} \subseteq V_{i_{0}}$, we have $\left|p_{0}\left(x_{i_{0}}\right)\right|<\varepsilon_{0}$, a contradiction. Q.E.D.

Definition 2.1. Let us denote $Q_{1}=\mathcal{Q}_{1}(A)$ a quasi-topology for $\operatorname{span}\left(\Phi_{A}\right)$ determined by quasi-neighbourhoods of the form

$$
U_{\varepsilon}=\left\{p \in \operatorname{span}\left(\Phi_{A}\right):\|p\|_{A^{*}}<\varepsilon\right\}
$$


where $\varepsilon>0$ and $\|\cdot\|_{A^{*}}$ denotes the norm in $A^{*}$.

Of course, $\mathcal{Q}_{1}$ is a local bases of the origin of the relative norm topology for $\operatorname{span}\left(\Phi_{A}\right)$, and hence $\mathcal{Q}_{0} \leq \mathcal{Q}_{1}$.

Lemma 2.2. The following three conditions for $A$ are equivalent:

(i) $\# \Phi_{A}<\infty$.

(ii) There exists a $\mathcal{Q}_{0}$-neighbourhood $U$ such that $\|p\|_{A^{*}} \leq 1$ for every $p \in U$.

(iii) $\mathcal{Q}_{0}(A) \cong \mathcal{Q}_{1}(A)$.

Proof. (i) $\Rightarrow$ (ii). Suppose that $\# \Phi_{A}<\infty$, and $\Phi_{A}=\left\{\varphi_{1}, \ldots, \varphi_{n}\right\}$. Since the hull-kernel topology on $\Phi_{A}$ is a $T_{1}$-topology, it follows that for any $i(1 \leq i \leq n)$,

\section{$\cap_{j: j \neq i} \operatorname{Ker} \varphi_{j} \nsubseteq \operatorname{Ker} \varphi_{i}$}

and so, there is an element $x_{i} \in A$ such that $\varphi_{j}\left(x_{i}\right)=\delta_{i j}(1 \leq j \leq n)$. Then every $p \in \operatorname{span}\left(\Phi_{A}\right)$ has a representation $p=\hat{p}\left(\varphi_{1}\right) \varphi_{1}+\ldots+\hat{p}\left(\varphi_{n}\right) \varphi_{n}$, and if we put $M=$ $\max _{1 \leq i \leq n}\left\|\varphi_{i}\right\|_{A^{*}}$, and consider a quasi-neighbourhood $U=U\left(x_{1}, \ldots, x_{n} ;(n M)^{-1}\right) \in \mathcal{Q}_{0}$, then a simple computation gives $\|p\|_{A^{*}} \leq 1$ for any $p \in U$.

(ii) $\Leftrightarrow$ (iii). Straightforward.

(ii) $\Rightarrow$ (i). Assume there is a quasi-neighbourhood $U=U\left(x_{1}, \ldots, x_{n} ; \varepsilon_{0}\right) \in \mathcal{Q}_{0}$ such that $\|p\|_{A^{*}} \leq 1(p \in U)$. Then we have to show that $\# \Phi_{A}<\infty$.

Suppose the contrary. Then we can take mutually distinct $n+1$ points $\varphi_{1}, \ldots, \varphi_{n+1}$ in $\Phi_{A}$. Choose a nontrivial vector $\left(a_{1}, \ldots, a_{n+1}\right) \in C^{n+1}$ such that

$$
a_{1} \varphi_{1}\left(x_{i}\right)+\ldots+a_{n+1} \varphi_{n+1}\left(x_{i}\right)=0
$$

for all $i=1, \ldots, n$. We can assume without loss of generality that $a_{1} \neq 0$. As observed in the proof of (i) $\Rightarrow$ (ii) above, we can choose an element $e \in A$ such that

$$
\|e\|=1, \varphi_{1}(e) \neq 0, \text { and } \varphi_{2}(e)=\ldots=\varphi_{n+1}(e)=0 .
$$

Let $c_{i}=2 a_{i} /\left(a_{1} \varphi_{1}(e)\right)$ for each $i=1, \ldots, n+1$ and set $p_{0}=c_{1} \varphi_{1}+\ldots+c_{n+1} \varphi_{n+1}$. Then $p_{0}\left(x_{i}\right)=0$ for all $i=1, \ldots, n$ and so $p_{0} \in U$, hence $\left\|p_{0}\right\|_{A^{*}} \leq 1$ by hypothesis . However, we have $\left\|p_{0}\right\|_{A^{*}} \geq\left|p_{0}(e)\right|=\left|c_{1} \varphi_{1}(e)\right|=2$, a contradiction. Q.E.D.

Remark 2.1. Let $L^{1}(G)$ be the group algebra of a LCA group $G$. Then, in order for $\mathcal{Q}_{0}\left(L^{1}(G)\right)<\mathcal{Q}_{1}\left(L^{1}(G)\right)$ to hold it is necessary and sufficient that $G$ is an infinite group by the preceding Lemma. 


\section{Induced algebras and Multiplier algebras}

Definition 3.1. We denote by $C_{B S E}\left(\Phi_{A}\right)$ the set of all continuous complex-valued functions $\sigma$ on $\Phi_{A}$ which satisfies the following: there exists a positive number $\beta$ such that

$$
\left|\sum_{\varphi \in \Phi_{A}} \hat{p}(\varphi) \sigma(\varphi)\right| \leq \beta\|p\|_{A^{*}}
$$

for every $p \in \operatorname{span}\left(\Phi_{A}\right)$. We put $\|\sigma\|_{B S E}$ the infimum of such $\beta$.

It is easy to see that $C_{B S E}\left(\Phi_{A}\right)=C\left(\Phi_{A} ; \mathcal{Q}_{1}\right)$ holds. $C_{B S E}\left(\Phi_{A}\right)$ becomes a semisimple Banach algebra under the pointwise multiplication and $\|\cdot\|_{B S E}$ norm (see [18, Lemma 1]). We call $C_{B S E}\left(\Phi_{A}\right)$ the induced algebra of $A$.

Remark 3.1. If $a \in A$, the Gelfand transform $\hat{a}$ of $a$ satisfies

$$
\left|\sum_{\varphi} \hat{p}(\varphi) \hat{a}(\varphi)\right| \leq\|a\|\|p\|_{A^{*}} \quad\left(p \in \operatorname{span}\left(\Phi_{A}\right)\right)
$$

and thus $\hat{a}$ belongs to $C_{B S E}\left(\Phi_{A}\right)$, and $\|\hat{a}\|_{B S E} \leq\|a\|(a \in A)$. Therefore $\hat{A}$ forms a subalgebra of $C_{B S E}\left(\Phi_{A}\right)$. As we will see later, in many algebras $A$ we consider, $\hat{A}$ is a closed subalgebra of $C_{B S E}\left(\Phi_{A}\right)$, but on the other hand, as we will see in $\S 8$, there are some algebras $A$ such that $\hat{A}$ are not closed in $C_{B S E}\left(\Phi_{A}\right)$.

A bounded linear operator $T$ of $A$ into itself is called a multiplier of $A$ if $x(T y)=$ $(T x) y$ holds for every $x, y \in A$. $M(A)$ denotes the set of all multipliers of $A . M(A)$ constitutes a commutative closed subalgebra of $\mathcal{B}(A)$, the Banach algebra of all bounded linear operators on $A$ into itself. It is well-known that each $T \in M(A)$ can be represented as a continuous function $\hat{T}$ on $\Phi_{A}$ such that $\hat{T} \hat{x}=\widehat{T x}(x \in A)$ (cf. $[6,8,20]$ ). We write $\hat{M}(A)=\{\hat{T}: T \in M(A)\}$.

An algebra $A$ is called a BSE-algebra if $\hat{M}(A)=C_{B S E}\left(\Phi_{A}\right)$ holds ([18]). Since the well-known Bochner-Schoenberg-Eberlein theorem for a LCA group $G$ can be restated as $\hat{M}\left(L^{1}(G)\right)=C_{B S E}(\hat{G})$, the group algebra $L^{1}(G)$ is a typical BSE-algebra (cf. [18, 19]).

Moreover, the second author and O. Hatori showed the following: 
Theorem C ([18]). The disk algebra $A(D)$, the classical Hardy algebra $H^{\infty}(D)$ and any commutative $C^{*}$-algebras are BSE.

Remark 3.2. It is easy to see that any unital BSE- algebra $A$ satisfies

$$
\hat{A}=C\left(\Phi_{A} ; \mathcal{Q}_{1}\right)=C_{B S E}\left(\Phi_{A}\right) .
$$

Therefore, $A(D)$ and $H^{\infty}(D)$ satisfies the condition (2). In addition, we see that any commutative $H^{*}$-algebra, $L^{p}(G)(1<p<\infty$, for compact $G), \ell^{1}(S)$ and the semigroup algebra $L^{1}\left(N_{k}\right)$, where $N_{k}=\{k, k+1, \ldots\}$ also satisfy the condition (2) from the results obtained in [19].

\section{Quasi-topologies $\mathcal{Q}_{D}$}

In this section, we consider quasi-topologies $\mathcal{Q}_{D}=\mathcal{Q}_{D}(A)$ for $A=C_{0}(\Omega)$.

Let $\Omega$ be a locally compact noncompact Hausdorff space, and let $A=C_{0}(\Omega)$ denote the commutative $C^{*}$-algebra consisting of all continuous functions on $\Omega$ which vanish at infinity. We can identify $\Phi_{C_{0}(\Omega)}$ with $\Omega$.

Definition 4.1. Let $\mathcal{Q}_{D}=\mathcal{Q}_{D}\left(C_{0}(\Omega)\right)$ be the family of subsets $V_{K, \delta}$ of $\operatorname{span}(\Omega)$ determined by the form

$$
V_{K, \delta}=\left\{p \in \operatorname{span}(\Omega):\|p\|_{C_{0}(\Omega)^{*}} \leq 1, \text { and } \int_{K} d|p|<\delta\right\}
$$

where $K$ is any compact subset of $\Omega, \varepsilon>0$ and $|p|$ denotes the total variation of a measure $p \in \operatorname{span}(\Omega)$. An easy computation shows that $\mathcal{Q}_{D}$ is a quasi- topology for $\operatorname{span}(\Omega)$. In this case, we have the following result.

Theorem 4.1. $C_{0}(\Omega)=C\left(\Omega ; \mathcal{Q}_{D}\right)$.

Proof. Let $0 \neq f \in C_{0}(\Omega)$ and $\varepsilon>0$. We set $K=\{\omega \in \Omega:|f(\omega)| \geq \varepsilon / 2\}$, and hence $K$ is compact. Set $\delta=\varepsilon /\left(2\|f\|_{\infty}\right)$, and let $p$ be any element of $V_{K, \delta}$. Then 
we can write $p=\sum_{\omega \in \Omega} \hat{p}(\omega) \delta_{\omega}$, and it is easy to see that $\|p\|_{C_{0}(\Omega)^{*}}=\sum_{\omega \in \Omega}|\hat{p}(\omega)|$. Therefore we have

$$
\begin{aligned}
\left|\sum_{\omega \in \Omega} \hat{p}(\omega) f(\omega)\right| & \leq \sum_{\omega \in K}\left|\hat{p}(\omega)\left\|f(\omega)\left|+\sum_{\omega \notin K}\right| \hat{p}(\omega)\right\| f(\omega)\right| \\
& \leq\|f\|_{\infty} \sum_{\omega \in K}|\hat{p}(\omega)|+(\varepsilon / 2) \sum_{\omega \notin K}|\hat{p}(\omega)| \\
& \leq\|f\|_{\infty} \int_{K} d|p|+(\varepsilon / 2)\|p\|_{C_{0}(\Omega)^{*}} \\
& \leq \varepsilon / 2+\varepsilon / 2=\varepsilon .
\end{aligned}
$$

Consequently, we conclude that $f \in C\left(\Omega ; \mathcal{Q}_{D}\right)$.

Conversely, let $f \in C\left(\Omega ; \mathcal{Q}_{D}\right)$ and $\varepsilon>0$. Then there exists a compact set $K$ and $\delta>0$ such that

$$
\left|\sum_{\omega \in \Omega} \hat{p}(\omega) f(\omega)\right|<\varepsilon / 2
$$

for all $p \in V_{K, \delta}$. Then if we set $K_{\varepsilon}=\{\omega \in \Omega: \mid f(\omega) \geq \varepsilon\}$, we have $K_{\varepsilon} \subseteq K$. Actually, if not, then there exists an element $\omega \in K_{\varepsilon} \backslash K$. Since $\omega \in K_{\varepsilon}$, we have $|f(\omega)| \geq \varepsilon$. But since $\omega \notin K$, and $\left\|\delta_{\omega}\right\|_{C_{0}(\Omega)^{*}}=1$, it follows that $\int_{K} d\left|\delta_{\omega}\right|=0(<\delta)$ and hence $\delta_{\omega} \in V_{K, \delta}$, so $|f(\omega)|<\varepsilon / 2$, a contradiction. Therefore $K_{\varepsilon}$ must be compact and so $f \in C_{0}(\Omega)$. Q.E.D.

\section{A quasi-topology $\mathcal{Q}_{c}$ and a local induced algebra $C_{B S E}^{0}\left(\Phi_{A}\right)$}

Definition 5.1. We denote by $\mathcal{Q}_{c}=\mathcal{Q}_{c}(A)$ the family of all the sets $U_{K, \delta}$ determined by

$$
\begin{array}{r}
U_{K, \delta}=\left\{p \in \operatorname{span}\left(\Phi_{A}\right):\|p\|_{A^{*}} \leq 1 \text { and } \exists q \in \operatorname{span}\left(\Phi_{A}\right)\right. \text { such that } \\
\left.\|q\|_{A^{*}} \leq \delta, \hat{p}(\varphi)=\hat{q}(\varphi)(\varphi \in K)\right\}
\end{array}
$$

where $K \in \mathcal{K}=\mathcal{K}\left(\Phi_{A}\right)$ : the set of all compact subsets of $\Phi_{A}$ and $\delta>0$. 
It is obvious that $\mathcal{Q}_{c}$ constitutes a quasi-topology for span $\left(\Phi_{A}\right)$. The introduction of $\mathcal{Q}_{c}$ is motivated by a wish to represent Theorem B in the context of the quasitopologies.

Definition 5.2. For $f \in C_{B S E}\left(\Phi_{A}\right)$ and $K \in \mathcal{K}$, we put

$$
\|f\|_{B S E, K}=\sup \left\{\left|\sum_{\varphi} \hat{p}(\varphi) f(\varphi)\right|: p \in \operatorname{span}\left(\Phi_{A}\right),\|p\|_{A^{*}} \leq 1, \hat{p}(\varphi)=0(\varphi \in K)\right\}
$$

and so we have $\|f\|_{B S E, K} \leq\|f\|_{B S E}$. Further, we set

$$
\begin{aligned}
\|f\|_{B S E, \infty} & =\inf _{K \in \mathcal{K}}\|f\|_{B S E, K}\left(=\lim _{K \in \mathcal{K}}\|f\|_{B S E, K}\right) \\
C_{B S E}^{0}\left(\Phi_{A}\right) & =\left\{f \in C_{B S E}\left(\Phi_{A}\right):\|f\|_{B S E, \infty}=0\right\} .
\end{aligned}
$$

It turns out that $C_{B S E}^{0}\left(\Phi_{A}\right)$ is a closed ideal of $C_{B S E}\left(\Phi_{A}\right)$ (Corollary 5.4). We call $C_{B S E}^{0}\left(\Phi_{A}\right)$ the local induced algebra of $A$.

Lemma 5.1. For each $f \in C_{B S E}\left(\Phi_{A}\right)$, the following equality holds:

$$
\|f\|_{B S E, \infty}=\lim _{U_{K, \delta} \in \mathcal{Q}_{c}} \sup \left\{\left|\sum_{\varphi} \hat{p}(\varphi) f(\varphi)\right|: p \in U_{K, \delta}\right\} .
$$

Proof. $\leq$ : For each $U_{K, \delta} \in \mathcal{Q}_{c}$ and $K_{1} \in \mathcal{K}$ with $K_{1} \supseteq K$,

" $p \in \operatorname{span}\left(\Phi_{A}\right),\|p\|_{A^{*}} \leq 1, \hat{p}(\varphi)=0\left(\varphi \in K_{1}\right)$ " imply " $p \in U_{K, \delta}$ ".

Therefore we have

$$
\|f\|_{B S E, K_{1}} \leq \sup \left\{\left|\sum_{\varphi} \hat{p}(\varphi) f(\varphi)\right|: p \in U_{K, \delta}\right\}
$$

and hence

$$
\|f\|_{B S E, \infty} \leq \sup \left\{\left|\sum_{\varphi} \hat{p}(\varphi) f(\varphi)\right|: p \in U_{K, \delta}\right\} .
$$

Taking the limit with respect to $U_{K, \delta}$ in $\mathcal{Q}_{c}$, we get the inequality " $\leq$ " in (3).

$\geq$ : Let $\varepsilon>0$ and $K_{1} \in \mathcal{K}$ be arbitrary, and choose a $\delta_{1}>0$ so that

$$
\left(1+\delta_{1}\right)\|f\|_{B S E, K_{1}}+\delta_{1}\|f\|_{B S E} \leq\|f\|_{B S E, K_{1}}+\varepsilon .
$$

For each $p \in U_{K_{1}, \delta_{1}}$, there exists a $q \in \operatorname{span}\left(\Phi_{A}\right)$ such that $\|q\|_{A^{*}} \leq \delta_{1}$ and $\hat{q}(\varphi)=$ $\hat{p}(\varphi)\left(\varphi \in K_{1}\right)$. Then we get

$$
\begin{aligned}
\left|\sum_{\varphi} \hat{p}(\varphi) f(\varphi)\right| & \leq\left|\sum_{\varphi}(\hat{p}(\varphi)-\hat{q}(\varphi)) f(\varphi)\right|+\left|\sum_{\varphi} \hat{q}(\varphi) f(\varphi)\right| \\
& \leq\left(1+\delta_{1}\right)\|f\|_{B S E, K_{1}}+\delta_{1}\|f\|_{B S E}
\end{aligned}
$$


By (4) and (5), we have

$$
\begin{aligned}
& \lim _{U_{K, \delta} \in \mathcal{Q}_{c}} \sup \left\{\left|\sum_{\varphi} \hat{p}(\varphi) f(\varphi)\right|: p \in U_{K, \delta}\right\} \\
& \quad \leq \sup \left\{\left|\sum_{\varphi} \hat{p}(\varphi) f(\varphi)\right|: p \in U_{K_{1}, \delta_{1}}\right\} \leq\|f\|_{B S E, K_{1}}+\varepsilon .
\end{aligned}
$$

Since $K_{1} \in \mathcal{K}$ and $\epsilon>0$ were arbitrary in (6), we get the the relation " $\geq$ " in (3). Q.E.D.

By Lemma 5.1, we get the following result.

Corollary 5.2. $C\left(\Phi_{A} ; \mathcal{Q}_{c}\right)=C_{B S E}^{0}\left(\Phi_{A}\right)$.

Lemma 5.3. For each $K \in \mathcal{K}$ and $f, g \in C_{B S E}\left(\Phi_{A}\right)$, we have

(i) $\|f g\|_{B S E, K} \leq\|f\|_{B S E, K} \cdot\|g\|_{B S E, K}$.

(ii) $\|f g\|_{B S E, \infty} \leq\|f\|_{B S E, \infty} \cdot\|g\|_{B S E, \infty}$.

Proof. We can prove (i) in the same way as that of Lemma 1 of [18], and we omit the proof. Taking the limit in (i) with respect to $K \in \mathcal{K}$, we get (ii). Q.E.D.

Corollary 5.4. $C_{B S E}^{0}\left(\Phi_{A}\right)$ is a closed ideal of $C_{B S E}\left(\Phi_{A}\right)$.

Proof. It is obvious that $C_{B S E}^{0}\left(\Phi_{A}\right)$ is an ideal of $C_{B S E}\left(\Phi_{A}\right)$ from Lemma 5.3 (ii). Let $\left\{f_{n}\right\}_{n \in N}$ be a Cauchy sequence in $C_{B S E}^{0}\left(\Phi_{A}\right)$ which converges to $f \in C_{B S E}\left(\Phi_{A}\right)$ in BSE-norm. Then,

$$
\|f\|_{B S E, \infty} \leq\left\|f-f_{n}\right\|_{B S E, \infty}+\left\|f_{n}\right\|_{B S E, \infty} \leq\left\|f-f_{n}\right\|_{B S E} \rightarrow 0(n \rightarrow \infty) .
$$

Thus we get $f \in C_{B S E}^{0}\left(\Phi_{A}\right)$, and $C_{B S E}^{0}\left(\Phi_{A}\right)$ is closed in $C_{B S E}\left(\Phi_{A}\right)$. Q.E.D. 


\section{Sufficient conditions for $\hat{A}=C_{B S E}^{0}\left(\Phi_{A}\right)$}

A commutative Banach algebra is called Tauberian if $A_{0}=\{x \in A: \hat{x}$ has a compact support $\}$ is norm dense in $A$ (cf. [9]).

Proposition 6.1. If $A$ is Tauberian, we have $\hat{A} \subseteq C_{B S E}^{0}\left(\Phi_{A}\right)$.

Proof. Obviously, we have $\hat{A}_{0} \subseteq C_{B S E}^{0}\left(\Phi_{A}\right)$. For each $x \in A$, choose $x_{n} \in A_{0} \quad(n=$ $1,2, \ldots)$ such that $\left\|x_{n}-x\right\| \rightarrow 0(n \rightarrow \infty)$, then we have

$$
\begin{aligned}
\|\hat{x}\|_{B S E, \infty} & \leq\left\|\hat{x}-\widehat{x_{n}}\right\|_{B S E, \infty}+\left\|\widehat{x_{n}}\right\|_{B S E, \infty} \\
& \leq\left\|x-x_{n}\right\| \rightarrow 0(n \rightarrow \infty),
\end{aligned}
$$

and hence $\hat{x} \in C_{B S E}^{0}\left(\Phi_{A}\right)$. Q.E.D.

Corollary 6.2. (i) If $A$ has a unit element, $\hat{A} \subseteq C_{B S E}^{0}\left(\Phi_{A}\right)=C_{B S E}\left(\Phi_{A}\right)$.

(ii) If $A$ is any one of the algebras $L^{p}(G)(1 \leq p<\infty, G$ : compact), commutative $H^{*}$-algebra, $\ell^{1}(S)$ (for any set), then $\hat{A}=C_{B S E}^{0}\left(\Phi_{A}\right)=C_{B S E}\left(\Phi_{\Phi_{A}}\right)$.

Proof. (i) is trivial.

(ii) In each case of $A, A$ is Tauberian and $\hat{A}=C_{B S E}\left(\Phi_{A}\right)$ (see [19]). Then (ii) follows from the preceding proposition. Q.E.D.

Proposition 6.3. $\hat{A}=C_{B S E}^{0}\left(\Phi_{A}\right)$ implies $C_{B S E}\left(\Phi_{A}\right) \subseteq \hat{M}(A)$.

Proof. By Corollary 5.4, $C_{B S E}^{0}\left(\Phi_{A}\right)$ is an ideal of $C_{B S E}\left(\Phi_{A}\right)$, and $\hat{A}=C_{B S E}^{0}\left(\Phi_{A}\right)$ implies that for each element $\sigma \in C_{B S E}\left(\Phi_{A}\right)$, the mapping $\hat{f} \rightarrow \hat{f} \sigma$ induces a linear operator of $\hat{A}$ into itself, which turns out to be a bounded operator in the induced norm $\|\hat{a}\|\|=\| a \|(a \in A)$ by the closed graph theorem. Therefore we get the relation $C_{B S E}\left(\Phi_{A}\right) \subseteq \hat{M}(A)$. Q.E.D. 
Proposition 6.4. Suppose that $A$ is a commutative semisimple regular and Tauberian Banach algebra. Further, we assume that $A$ has an approximate identity $\left\{e_{\lambda}\right\}_{\lambda \in \Lambda}$ such that $\sup \left\{\left\|M_{e_{\lambda}}\right\|: \lambda \in \Lambda\right\}=C<\infty$, where $\left\|M_{e_{\lambda}}\right\|$ denotes the operator norm of the multiplication operator $M_{e_{\lambda}}$ on $A$ induced by $e_{\lambda}$. Then, if $C_{B S E}\left(\Phi_{A}\right) \subseteq \hat{M}(A), \hat{A}$ is $\|\cdot\|_{B S E}-$ norm dense in $C_{B S E}^{0}\left(\Phi_{A}\right)$. Moreover, if $\left\{e_{\lambda}\right\}_{\lambda \in \Lambda}$ is bounded in the norm of $A$, then we have $\hat{A}=C_{B S E}^{0}\left(\Phi_{A}\right)$.

Remark 6.1. If $\left\{e_{\lambda}\right\}_{\lambda \in \Lambda}$ is a bounded approximate identity, then it is clear that $\sup \left\{\left\|M_{e_{\lambda}}\right\|: \lambda \in \Lambda\right\}<\infty$. Also, if an approximate identity is of the form $\left\{e_{n}\right\}_{n \in N}$, then we have $\sup \left\{\left\|M_{e_{n}}\right\|: n \in N\right\}<\infty$ by the Banach-Steinhaus theorem. Thus $\left\{e_{n}\right\}_{n \in N}$ satisfies the condition required in Proposition 6.4.

For the proof of Proposition 6.4, we need the next Lemma.

Lemma 6.5. For $a, b \in A$ and $p \in \operatorname{span}\left(\Phi_{A}\right)$, define elements $p+\hat{a} p, \hat{a} p+\hat{b} p \in$ $\operatorname{span}\left(\Phi_{A}\right) b y$

$$
\begin{aligned}
(p+\hat{a} p)^{\wedge}(\varphi) & =\hat{p}(\varphi)+\hat{a}(\varphi) \hat{p}(\varphi), \\
(\hat{a} p+\hat{b} p)^{\wedge}(\varphi) & =\hat{a}(\varphi) \hat{p}(\varphi)+\hat{b}(\varphi) \hat{p}(\varphi)
\end{aligned}
$$

for each $\varphi \in \Phi_{A}$, then we have the following inequalities:

(i) $\|p+\hat{a} p\|_{A^{*}} \leq\left(1+\left\|M_{a}\right\|\right)\|p\|_{A^{*}} \leq(1+\|a\|)\|p\|_{A^{*}}$.

(ii) $\|\hat{a} p+\hat{b} p\|_{A^{*}} \leq\left\|M_{a+b}\right\|\|p\|_{A^{*}} \leq\|a+b\|\|p\|_{A^{*}}$.

$$
\text { Proof. (i) } \begin{aligned}
\| p & +\hat{a} p \|_{A^{*}}=\sup \left\{\left|\sum_{\varphi}(\hat{p}(\varphi)+\hat{a}(\varphi) \hat{p}(\varphi)) \hat{x}(\varphi)\right|: x \in A,\|x\| \leq 1\right\} \\
& \leq \sup \left\{\left|\sum_{\varphi} \hat{p}(\varphi) \hat{x}(\varphi)\right|+\left|\sum_{\varphi} \hat{p}(\varphi) \widehat{a x}(\varphi)\right|: x \in A,\|x\| \leq 1\right\} \\
& \leq \sup \left\{\|p\|_{A^{*}}\|x\|+\|p\|_{A^{*}}\|a x\|: x \in A,\|x\| \leq 1\right\} \\
& \leq\left(1+\left\|M_{a}\right\|\right)\|p\|_{A^{*}} \leq(1+\|a\|)\|p\|_{A^{*}} .
\end{aligned}
$$

(ii) $\|\hat{a} p+\hat{b} p\|_{A^{*}}=\sup \left\{\left|\sum_{\varphi}(\hat{a}(\varphi) \hat{p}(\varphi)+\hat{b}(\varphi) \hat{p}(\varphi)) \hat{x}(\varphi)\right|: x \in A,\|x\| \leq 1\right\}$ 


$$
\begin{aligned}
& \leq \sup \left\{\mid \sum_{\varphi} \hat{p}(\varphi)((a+b) x)^{\wedge}(\varphi)\|: x \in A,\| x \| \leq 1\right\} \\
& \leq \sup \left\{\|p\|\left\|_{A^{*}}\right\|(a+b) x\|: x \in A,\| x \| \leq 1\right\} \\
& \leq\left\|M_{a+b}\right\|\|p\|_{A^{*}} \leq\|a+b\|\|p\|_{A^{*}} \text {. Q.E.D. }
\end{aligned}
$$

Proof of Proposition 6.4. Suppose $f \in C_{B S E}^{0}\left(\Phi_{A}\right)$, and let $\varepsilon>0$ be arbitrary. Then, by Corollary 5.2, there exist $K \in \mathcal{K}=\mathcal{K}\left(\Phi_{A}\right)$ and $\delta(\varepsilon>\delta>0)$ such that

$$
\sup \left\{\left|\sum_{\varphi} \hat{p}(\varphi) f(\varphi)\right|: p \in U_{K, \delta}\right\}<\varepsilon .
$$

Since $A$ is regular, we can choose $e_{K} \in A$ such that $\widehat{e_{K}}(\varphi)=1(\varphi \in K)$. Then there exist $\lambda_{1} \in \Lambda$ and $\lambda_{2} \in \Lambda$ such that

$$
\begin{aligned}
\left\|e_{K} e_{\lambda_{1}}-e_{K}\right\| & <\delta \\
\left\|e_{\lambda_{1}} e_{\lambda_{2}}-e_{\lambda_{1}}\right\| & <\delta .
\end{aligned}
$$

We put $g=f-f \cdot \widehat{e_{\lambda_{2}}} \in C_{B S E}^{0}\left(\Phi_{A}\right)$. Since $f \cdot \widehat{e_{\lambda_{2}}}$ belongs to $\hat{A}$ by the assumption $C_{B S E}\left(\Phi_{A}\right) \subseteq \hat{M}(A)$, we can prove the first assertion of the theorem by estimating the $B S E$-norm of $g$.

Let $p \in \operatorname{span}\left(\Phi_{A}\right)$ be arbitrarily chosen such that $\|p\|_{A^{*}} \leq 1$. Then

$$
\left|\sum_{\varphi} \hat{p}(\varphi) g(\varphi)\right| \leq\left|\sum_{\varphi}\left(\hat{p}(\varphi)-\hat{p}(\varphi) \widehat{e_{\lambda_{1}}}(\varphi)\right) g(\varphi)\right|+\left|\sum_{\varphi} \hat{p}(\varphi) \widehat{e_{\lambda_{1}}}(\varphi) g(\varphi)\right|
$$

By Lemma $6.5,\left\|p-p \widehat{e_{\lambda_{1}}}\right\|_{A^{*}} \leq 1+C$, and if we put $t=\frac{1}{1+C}\left(p-\widehat{p} \widehat{e_{\lambda 1}}\right)$, then $t \in \operatorname{span}\left(\Phi_{A}\right)$ and $\|t\|_{A^{*}} \leq 1$. Also, if we put

$$
q=t \widehat{e_{K}}=\frac{1}{1+C}\left(p \widehat{e_{K}}-p \widehat{e_{\lambda_{1}}} \widehat{e_{K}}\right) \in \operatorname{span}\left(\Phi_{A}\right)
$$

we get by Lemma 6.5 and ( 8 )

$$
\begin{aligned}
\|q\|_{A^{*}} & =\frac{1}{1+C}\left\|p \widehat{e_{K}}-\widehat{p} \widehat{e_{\lambda_{1}}} \widehat{e_{K}}\right\|_{A^{*}} \\
& \leq \frac{1}{1+C}\left\|e_{K}-e_{K} e_{\lambda_{1}}\right\| p \|_{A^{*}} \leq \frac{\delta}{1+C}<\delta,
\end{aligned}
$$

and $\hat{q}(\varphi)=\hat{t}(\varphi) \widehat{e_{K}}(\varphi)=\hat{t}(\varphi)(\varphi \in K)$. Thus $t \in U_{K, \delta}$. In the same way, we have $t_{1}=\frac{1}{1+C} t \widehat{t \lambda_{2}} \in U_{K, \delta}$. In fact, by Lemma 6.5 we have

$$
\left\|t_{1}\right\|_{A^{*}} \leq \frac{1}{1+C}\|t\|_{A^{*}}\left\|M_{e_{\lambda_{2}}}\right\| \leq \frac{C}{1+C} \leq 1,
$$


and if we put $q_{1}=t_{1} \widehat{e_{K}}$, we have

$$
\begin{aligned}
\left\|q_{1}\right\|_{A^{*}} & =\left\|\frac{1}{1+C}\left(\frac{1}{1+C}\left(p-p \cdot \widehat{e_{\lambda_{1}}}\right)\right) \widehat{e_{\lambda_{2}} e_{K}}\right\|_{A^{*}} \\
& \leq \frac{1}{(1+C)^{2}}\|p\|_{A^{*}}\left\|\left(e_{K}-e_{\lambda_{1}} e_{K}\right) e_{\lambda_{2}}\right\| \\
& \leq \frac{1}{(1+C)^{2}}\left\|e_{K}-e_{\lambda_{1}} e_{K}\right\|\left\|M_{e_{\lambda_{2}}}\right\| \leq \frac{C \delta}{(1+C)^{2}}<\delta
\end{aligned}
$$

and $q_{1}(\varphi)=t_{1}(\varphi) \quad(\varphi \in K)$.

Consequently, we get $t, t_{1} \in U_{K, \delta}$. Hence by (7), we can estimate the right hand side of (10) as follows:

$$
\begin{aligned}
\mid \sum_{\varphi}(\hat{p}(\varphi) & \left.-\hat{p}(\varphi) \widehat{e_{\lambda_{1}}}(\varphi)\right) g(\varphi) \mid \\
& =\left|\sum_{\varphi}\left(\hat{p}(\varphi)-\hat{p}(\varphi) \widehat{e_{\lambda_{1}}}(\varphi)\right) f(\varphi)-\sum_{\varphi}\left(\hat{p}(\varphi)-\hat{p}(\varphi) \widehat{e_{\lambda_{1}}}(\varphi)\right) f(\varphi) \widehat{e_{\lambda_{2}}}(\varphi)\right| \\
& \leq(1+C)\left|\sum_{\varphi} \hat{t}(\varphi) f(\varphi)\right|+(1+C)^{2}\left|\sum_{\varphi} \widehat{t_{1}}(\varphi) f(\varphi)\right| \\
& \leq(1+C) \varepsilon+(1+C)^{2} \varepsilon=(1+C)(2+C) \varepsilon
\end{aligned}
$$

and by (9)

$$
\begin{aligned}
\left|\sum_{\varphi} \hat{p}(\varphi) \widehat{e_{\lambda_{1}}}(\varphi) g(\varphi)\right| & =\left|\sum_{\varphi}\left(\hat{p}(\varphi) \widehat{e_{\lambda_{1}}}(\varphi)-\hat{p}(\varphi) \widehat{e_{\lambda_{1}}}(\varphi) \widehat{e_{\lambda_{2}}}(\varphi)\right) f(\varphi)\right| \\
& \leq\|p\|_{A^{*}}\left\|\left(\widehat{e_{\lambda_{1}}}-\widehat{e_{\lambda_{1}} e_{\lambda_{2}}}\right) f\right\|_{B S E} \leq\left\|\widehat{e_{\lambda_{1}}}-\widehat{e_{\lambda_{1}} e_{\lambda_{2}}}\right\|_{B S E}\|f\|_{B S E} \\
& \leq\left\|e_{\lambda_{1}}-e_{\lambda_{1}} e_{\lambda_{2}}\right\|\|f\|_{B S E} \leq \delta\|f\|_{B S E} \leq \varepsilon\|f\|_{B S E}
\end{aligned}
$$

By (10), (11) and (12) we get

$$
\begin{aligned}
\|g\|_{B S E} & =\sup \left\{\left|\sum_{\varphi} \hat{p}(\varphi) \hat{g}(\varphi)\right|: p \in \operatorname{span}\left(\Phi_{A}\right),\|p\|_{A^{*}} \leq 1\right\} \\
& \leq\left((1+C)(1+2 C)+\|f\|_{B S E}\right) \varepsilon .
\end{aligned}
$$

Since $\varepsilon>0$ was arbitrary, $f$ can be approximated by elements of $\hat{A}$ in BSE-norm.

Finally, suppose $A$ has a bounded approximate identity $\left\{e_{\lambda}\right\}_{\lambda \in \Lambda}$ with $\left\|e_{\lambda}\right\| \leq C<$ $\infty(\lambda \in \Lambda)$. In this case, we note the following (a) and (b):

(a) $\hat{M}(A) \subseteq C_{B S E}\left(\Phi_{A}\right)$ by Corollary 5 of [18]. This fact with the assumption $C_{B S E}\left(\Phi_{A}\right) \subseteq \hat{M}(A)$ implies that $A$ is BSE, and hence BSE-norm on $C_{B S E}\left(\Phi_{A}\right)$ is equivalent to the multiplier operator norm by Corollary 6 of [18].

(b) $\left\|M_{a}\right\|=\sup _{x \in A, 0<\|x\| \leq 1}\|a \cdot x\| \geq \sup _{\lambda \in \Lambda}\left\|a \cdot e_{\lambda} / C\right\| \geq\|a\| / C \quad(a \in A)$. 
It follows from (a) and (b) that the BSE-norm of $\hat{A}$ is a complete norm, which implies $C_{B S E}^{0}\left(\Phi_{A}\right) \subseteq \hat{A}$ since each $f \in C_{B S E}^{0}\left(\Phi_{A}\right)$ is a BSE-norm limit of a sequence of elements in $\hat{A}$ as we have shown above. Since $A$ is Tauberian, the reverse inclusion follows from Proposition 6.1, and thus we get $C_{B S E}^{0}\left(\Phi_{A}\right)=\hat{A}$. Q.E.D.

Theorem 6.6. Let $A$ be a commutative semisimple regular and Tauberian Banach algebra with a bounded approximate identity $\left\{e_{\lambda}\right\}_{\lambda}$. Then the following two conditions are equivalent:

(i) $\hat{A}=C_{B S E}^{0}\left(\Phi_{A}\right)$.

(ii) $A$ is a BSE-algebra, i.e., $\hat{M}(A)=C_{B S E}\left(\Phi_{A}\right)$.

Proof. (i) $\Rightarrow$ (ii) Since $A$ has a bounded approximate identity, we have $\hat{M}(A) \subseteq$ $C_{B S E}\left(\Phi_{A}\right)$ (cf. Corollary 5 of [18]), and the reverse inclusion follows from Proposition 6.3.

(ii) $\Rightarrow$ (i) Since $A$ is BSE, we have $C_{B S E}\left(\Phi_{A}\right) \subseteq \hat{M}(A)$, and since $A$ has a bounded approximate identity, (i) follows at once from Proposition 6.4. Q.E.D.

The next corollary is essentially a restatement of Theorem B.

Corollary 6.7. $L^{1}(G)^{\wedge}=C_{B S E}^{0}\left(\Phi_{L^{1}(G)}\right)=C\left(\Phi_{L^{1}(G)}, \mathcal{Q}_{c}\right)$.

Proof. It is well-known that $L^{1}(G)$ is a commutative Banach algebra which is semisimple, regular, Tauberian with a bounded approximate identity. Moreover, $L^{1}(G)$ is BSE (see [18]) by the Bochner-Schoenberg-Eberlein theorem. Therefore the conclusion of the corollary follows at once from Theorem 6.6 and Corollary 5.2. Q.E.D. 


\section{Algebras which satisfy $\hat{A}=C_{B S E}^{0}\left(\Phi_{A}\right)$}

In this section, we study some concrete Banach algebras $A$ which satisfy $\hat{A}=$ $C_{B S E}^{0}\left(\Phi_{A}\right)$.

For a LCA group $G, M(G)$ denotes the space of all the bounded regular complex measures on $G$. For each $\mu \in M(G), \hat{\mu}$ expresses the Foureir-Stieltjes transform of $\mu$. For a Haar measurable subset $E$ of $\hat{G}$, we use the following notations:

$M_{1}(E)=\{\mu:$ a discrete measure whose support is a finite set contained in $E\}$.

$I_{0}(E)=\left\{f \in L^{1}(G): \hat{f}\right.$ vanishes on a neighbourhood of $\left.E\right\}$.

$I_{00}(E)=\left\{f \in I_{0}(E): \hat{f}\right.$ has a compact support in $\left.\hat{G} \backslash E\right\}$.

$I(E)=\left\{f \in L^{1}(G): \hat{f}\right.$ vanishes on $\left.E\right\}$.

The following result is known.

Theorem D ([13], [11]). Let $f$ be a Haar-measurable complex function defined on a Haar measurable set $E \subseteq \hat{G}$. If there exists a constant $C$ such that

$$
\left|\int f d \mu\right| \leq C\|\hat{\mu}\|_{\infty}\left(\mu \in M_{1}(E)\right)
$$

there exists $a \nu \in M(G)$ such that $\|\nu\| \leq C$ and $\hat{\nu}=f$ a.e. on $E$.

Lemma 7.1. Let $E$ be a closed subset of $\hat{G}$. Then in both cases $A=L^{1}(G) / I(E)$ and $A=I(E)$, we have $C_{B S E}\left(\Phi_{A}\right) \subseteq \hat{M}(A)$.

Proof. We remark first that in the case of $A=I(E), \Phi_{A}$ can be naturally identified with $\hat{G} \backslash E$, and in the case of $A=L^{1}(G) / I(E), \Phi_{A}$ can be naturally identified with $E$. Therefore each $p \in \operatorname{span}\left(\Phi_{A}\right)$ can be expressed in the form $p(x)=\sum_{\gamma \in F} \hat{p}(\gamma)(-x, \gamma)$, where $F=\hat{G} \backslash E$ in the case of $A=I(E)$ and $F=E$ in the case of $A=L^{1}(G) / I(E)$.

Let $\sigma \in C_{B S E}\left(\Phi_{A}\right)$ be arbitrary. Note that for each $p \in \operatorname{span}\left(\Phi_{A}\right)$ we have,

(i) in the case of $A=I(E)$ :

$$
\begin{aligned}
\|p\|_{A^{*}} & =\sup \left\{\left|\sum_{\gamma \in F} \hat{p}(\gamma) \hat{f}(\gamma)\right|: f \in I(E),\|f\|_{1} \leq 1\right\} \\
& \leq \sup \left\{\left|\sum_{\gamma \in F} \hat{p}(\gamma) \hat{f}(\gamma)\right|: f \in L^{1}(G),\|f\|_{1} \leq 1\right\}=\|p\|_{\infty} .
\end{aligned}
$$


(ii) in the case $A=L^{1}(G) / I(E)$ :

$$
\begin{aligned}
\|p\|_{A^{*}} & =\sup \left\{\left|\sum_{\gamma \in F} \hat{p}(\gamma) \hat{f}(\gamma)\right|:[f] \in L^{1}(G) / I(E),\|[f]\|_{L^{1}(G) / I(E)} \leq 1\right\} \\
& =\sup \left\{\left|\sum_{\gamma \in F} \hat{p}(\gamma) \hat{f}(\gamma)\right|: f \in L^{1}(G),\|f\|_{1} \leq 1\right\}=\|p\|_{\infty} .
\end{aligned}
$$

Therefore we get for each $p \in \operatorname{span}(F)$,

$$
\left|\sum_{\gamma \in F} \hat{p}(\gamma) \sigma(\gamma)\right| \leq\|\sigma\|_{B S E}\|\dot{p}\|_{A^{*}} \leq\|\sigma\|_{B S E}\|\| p \|_{\infty} .
$$

Obviously, (13) is equivalent to

$$
\left|\int \sigma d \mu\right| \leq\|\sigma\|_{B S E}\|\hat{\mu}\|_{\infty} \quad\left(\mu \in M_{1}(F)\right) .
$$

By Theorem D, (14) implies that there exists a $\nu \in M(G)$ such that $\left.\hat{\nu}\right|_{F}=\sigma,\|\nu\| \leq$ $\|\sigma\|_{B S E}$. Therefore, it follows easily that $\sigma$ belongs to $\hat{M}(A)$ in each case of $A=I(E)$ and $A=L^{1}(G) / I(E)$. Q.E.D.

Theorem 7.2. For each closed subset $F$ of $\hat{G}$, the quotient algebra $A=L^{1}(G) / I(F)$ is $\mathrm{BSE}$, and satisfies

$$
\hat{A}=C_{B S E}^{0}\left(\Phi_{A}\right)=C\left(\Phi_{A} ; \mathcal{Q}_{c}\right) .
$$

Proof. Obviously, $A$ has a bounded approximate identity, and hence we have $\hat{M}(A) \subseteq C_{B S E}\left(\Phi_{A}\right)$ by Corollary 5 of $[18]$.

Conversely, $\hat{M}(A) \supseteq C_{B S E}\left(\Phi_{A}\right)$ follows from Lemma 7.1, and hence $A$ is BSE. Therefore the first equality in (15) follows from Theorem 6.6 since $A$ is a commutative semisimple regular and Tauberian Banach algebra with a bounded approximate identity. The second equality in (15) follows from Corollary 5.2. Q.E.D.

We note that Theorem 7.2 is a generalization of Corollary 9 of [18]. Next, we consider the problem under what conditions the closed ideals $I(E)$ of $L^{1}(G)$ are BSE (cf. $[18]$ ).

This problem is closely related with the strong Ditkin sets. For the definition of the strong Ditkin sets, we follow Saeki [17].

Definition 7.1. A closed subset $E$ of $\hat{G}$ is called a strong Ditkin set if there exists a family $\left\{\mu_{\lambda} \in M(G)\right\}_{\lambda \in \Lambda}$ of measures which is directed, in the sense that the index set $\Lambda$ is a directed set such that 
(a) $\sup \left\{\left\|\mu_{\lambda}\right\|_{E}: \lambda \in \Lambda\right\}=C<\infty$, where $\left\|\mu_{\lambda}\right\|_{E}=\sup \left\{\left\|f * \mu_{\lambda}\right\|_{1}: f \in\right.$ $\left.I_{0}(E),\|f\|_{1} \leq 1\right\}$,

(b) $\widehat{\mu_{\lambda}}=0$ on some neighbourhood of $E$ depending on $\mu_{\lambda}(\lambda \in \Lambda)$,

(c) $\lim _{\lambda \in \Lambda}\left\|f * \mu_{\lambda}-f\right\|_{1}=0 \quad(f \in I(E))$.

Definition 7.2. We denote by $\Sigma_{d}(\hat{G})$ the discrete coset-ring of $\hat{G}$, i.e., the Boolean algebra generated by the cosets of all subgroups (whether closed or not) of $\hat{G}$.

Lemma 7.3. Let $E$ be a closed subset of $\hat{G}$, and suppose there exists a deirected set of measures $\left\{\mu_{\lambda} \in M(G)\right\}_{\mu \in \Lambda}$ which satisfies conditions (a), (b) and (c) of Definition 7.1. Then we can construct a dircted set of functions in $I_{00}(E)$ which satisfies conditions (a), (b) and (c) of Definition 7.1. Moreover, if $\left\{\mu_{\lambda}\right\}_{\mu \in \Lambda}$ is norm bounded, then we can also construct it as norm bounded.

Proof. We first remark that under the conditions (b) and (c) in Definition 7.1, $I_{0}(E)$ is norm dense in $I(E)$. Therefore we have

$$
\left\|\mu_{\lambda}\right\|_{E}=\sup \left\{\left\|f * \mu_{\lambda}\right\|_{1}: f \in I(E),\|f\|_{1} \leq 1\right\} \quad(\lambda \in \Lambda) .
$$

Let $\left\{u_{\beta}\right\}_{\beta \in B}$ be an approximate identity of $L^{1}(G)$ such that $\hat{u}_{\beta}$ has a compact support and $\left\|u_{\beta}\right\|_{1}=1$ for each $\beta \in B$. Then $\left\{\mu_{\lambda} * u_{\beta}\right\}_{(\lambda, \beta) \in \Lambda \times B}$ is a net in $I_{00}(E)$ with an index set $\Lambda \times B$. That $\left\{\mu_{\lambda} * u_{\beta}\right\}_{(\lambda, \beta) \in \Lambda \times B}$ satisfies (a) and (b) of Definition 7.1 is obvious. To show that (c) is also satisfied, let $f \in I(E)$ and $\varepsilon>0$ be arbitrarily chosen. Then by (c), there exists $\lambda_{0} \in \Lambda$ such that $\left\|f-f * \mu_{\lambda}\right\|_{1} \leq \varepsilon / 2\left(\lambda \in \Lambda, \lambda_{0} \leq \lambda\right)$. Choose $\beta_{0} \in B$ such that $\left\|u_{\beta} * f-f\right\|_{1} \leq \varepsilon / 2 C\left(\beta \geq \beta_{0}\right)$. Then by (16) we have

$$
\begin{aligned}
\left\|\mu_{\lambda} * u_{\beta} * f-f\right\|_{1} & \leq\left\|\mu_{\lambda} *\left(u_{\beta} * f-f\right)\right\|_{1}+\left\|\mu_{\lambda} * f-f\right\|_{1} \\
& \leq \sup \left\{\left\|\mu_{\lambda} * g\right\|_{1}: g \in I(E),\|g\|_{1} \leq \varepsilon /(2 C)\right\}+\varepsilon / 2 \\
& =\left\|\mu_{\lambda}\right\|_{E} \cdot \varepsilon /(2 C)+\varepsilon / 2 \\
& \leq C \cdot(\varepsilon / 2 C)+\varepsilon / 2=\varepsilon \quad\left(\lambda_{0} \leq \lambda, \beta_{0} \leq \beta\right) .
\end{aligned}
$$

Thus $\left\{\mu_{\lambda} * u_{\beta}\right\}_{(\Lambda, \beta) \in \Lambda \times B}$ satisfies (c). Moreover it is easy to see that, if $\left\{\mu_{\lambda}\right\}_{\lambda \in \Lambda}$ is norm bounded in $M(G)$, then $\left\{\mu_{\lambda} * u_{\beta}\right\}_{(\Lambda, \beta) \in \Lambda \times B}$ is also norm bounded in $I_{00}(E)$. Q.E.D.

To proceed our study, we need the next lemma, which is a generalization of Theorem 1 of Gilbert [4]. 
Lemma 7.4.(Saeki [17]) If $E$ is a closed subset of $\hat{G}$ which belongs to $\Sigma_{d}(\hat{G}), E$ has a norm bounded net $\left\{\mu_{\lambda}\right\}_{\lambda \in \Lambda} \subseteq M(G)$ which satisfies the conditions (b) and (c) of Definition 7.1, and hence $E$ is a strong Ditkin set.

The proof of Lemma 7.4 is contained in the proof of "(iii) $\Rightarrow$ (i)" in Theorem 7 of [17]. (Thoerem 7 of [17] is stated under the assumption that $E$ has no interier points, but its proof of "(iii) $\Rightarrow$ (i)" is valid without this assumption).

Theorem 7.5. Suppose that $E$ is a closed subset of $\hat{G}$. Then the following two conditions are equivalent:

(i) $I(E)$ is BSE.

(ii) $E$ is an element of $\Sigma_{d}(\hat{G})$.

Proof. (i) $\Rightarrow$ (ii) Suppose that $I(E)$ is BSE. Then by Corollary 5 of $[18], I(E)$ has a bounded weak approximate identity $\left\{u_{\lambda}\right\}_{\lambda \in \Lambda}$ in the sense of Jones-Lahr: that is, $\sup \left\{\left\|u_{\lambda}\right\|_{1}: \lambda \in \Lambda\right\}=C<\infty$, and $\lim _{\lambda} \widehat{u}_{\lambda}(\gamma) \hat{f}(\gamma)=\hat{f}(\gamma)(f \in I(E), \gamma \in \hat{G} \backslash E)$. We may regard each $u_{\lambda}$ as defining a finite measure, say $\mu_{\lambda}$ on $\bar{G}$, the Bohr compactification of $G$. By the compactness of the $C$-ball of a conjugate space in the weak ${ }^{*}$-topology and the Riesz representation theorem, there exists a finite measure $\mu$ on $\bar{G}$ which is a limit point of some subnet of $\left\{\mu_{\lambda}: \lambda \in \Lambda\right\}$ in the weak*- topology of $M(\bar{G})$. From this we can conclude that

$$
\hat{\mu}(\gamma)= \begin{cases}0 & (\gamma \in E), \\ 1 & (\gamma \in \widehat{G} \backslash E) .\end{cases}
$$

Thus $\mu$ is an idempotent measure in $M(\bar{G})$, and by Cohen's idempotent theorem ([1], [16]), $E$ belongs to $\Sigma_{d}(\hat{G})$ (cf. the proof of Theorem 1.1 [14]).

(ii) $\Rightarrow$ (i) Suppose that $E \in \Sigma_{d}(\hat{G})$. Then by Lemmas 7.3 and 7.4 , it follows that $I(E)$ has a bounded approximate identity consisting of functions in $I_{00}(E)$. Therefore by Corollary 5 of [18], we have $\hat{M}(I(E)) \subseteq C_{B S E}\left(\Phi_{I(E)}\right)$. The reverse inclusion follows from Lemma 7.1, and we get $C_{B S E}\left(\Phi_{I(E)}\right)=\hat{M}(I(E))$. Q.E.D.

Corollary 7.6. If $E$ is a closed subset of $\hat{G}$ which belongs to $\Sigma_{d}(\hat{G})$, then

$$
I(E)^{\wedge}=C_{B S E}^{0}\left(\Phi_{I(E)}\right)
$$

Proof. As we have shown in the proof of Theorem 7.5, $I(E)$ has a bounded approximate identity consisting of functions in $I_{00}(E)$, which implies that $I(E)$ is Tauberian. 
Of course $I(E)$ is a commutative semisimple, regular Banach algebra, and so we get $I(E)^{\wedge}=C_{B S E}^{0}\left(\Phi_{I(E)}\right)$ by Theorem 7.5 and Theorem 6.6. Q.E.D.

Remark 7.1. When $E=\emptyset$, Theorem 7.5 (ii) $\Rightarrow$ (i) reduces to the BochnerSchoenberg-Eberlein theorem (BSE-theorem) and Corollary 7.6 reduces to Theorem B. Therefore Theorem 7.5 and Corollary 7.6 can be seen as a generalization of BSEtheorem and Theorem B, respectively.

Theorem 7.7. Any commutative $C^{*}$-algebra A satisfies $\hat{A}=C_{B S E}^{0}\left(\Phi_{A}\right)$.

Proof. Obviously, $A$ satisfies all the conditions for $A$ in Theorem 6.6. Moreover $A$ is BSE (Theorom C), and hence we get the desierd conclusion by Theorem 6.6. Q.E.D.

Remark 7.2. It is easy to see that Theorem 7.7 can be proved easily by direct calculations. 


\section{Algebras which satisfy $\hat{A} \neq C_{B S E}^{0}\left(\Phi_{A}\right)$}

In this section, we investigate some Banach algebras $A$ which satisfy $\hat{A} \neq C_{B S E}^{0}\left(\Phi_{A}\right)$.

At first, let us consider the Segal algebra $S^{p}(G)$ for a noncompact locally compact abelian group $G$.

For a noncompact locally compact abelian group $G$ and $p(1<p<\infty)$, let $S^{p}(G)=$ $L^{1}(G) \cap L^{p}(G)$, and define a norm in $S^{p}(G)$ by

$$
\|f\|_{s}=\max \left\{\|f\|_{1},\|f\|_{p}\right\} \quad\left(f \in S^{p}(G)\right) .
$$

It is known that $S^{p}(G)$ become a commutative regular semisimple Banach algebra with the convolution multiplication (cf. [10] Theorem 6), and the Gelfand space can be identified with $\hat{G} . S^{p}(G)$ belongs to the class of a Banach algebra called Segal algebras (cf. [12], [21]).

We will show that $S^{p}(G)$ is a BSE-algebra, and that the relation $S^{p}(G)^{\wedge} \subset_{\neq} C_{0}\left(\Phi_{S^{p}(G)}\right)$ holds. The proof of the following lemma is due to [5].

Proposition 8.1. Let $G$ be a non-compact locally compact abelian group, and $\hat{G}$ be the dual group of $G$. Suppose $1<q<\infty$, and $0 \neq f \in \operatorname{span}(\hat{G})$. Then we have $\|f-g\|_{\infty} \geq\|f\|_{\infty}$ for each $g \in L^{q}(G)$.

Proof. Suppose that the proposition is not true, that is, there exists $g \in L^{q}(G)$ and $z_{0} \in G$ such that $\|f-g\|_{\infty}<\left|f\left(z_{0}\right)\right|$. We put

$$
\varepsilon:=\frac{1}{3}\left(\left|f\left(z_{0}\right)\right|-\|f-g\|_{\infty}\right)>0
$$

and we will get a contradiction.

Since $f$ is an almost periodic function on $G$, there exists a finite set $F \subset G$ such that; for each $y \in G$, we can choose $\tau(y) \in F$ so that $\left\|f_{y}-f_{\tau(y)}\right\|_{\infty}<\varepsilon$, where $f_{y}$ denote the translation of $f$ by $y, f_{y}(x)=f(x-y)$. By the continuity of $f$, we can choose a compact neighbourhood $K_{0}$ of $z_{0}$ such that

$$
|f(x)| \geq\left|f\left(z_{0}\right)\right|-\varepsilon \quad\left(x \in K_{0}\right)
$$


Here we define a compact set $K$ by $K=K_{0}-K_{0}+F-F$. Since $G$ is non-compact, we can choose a sequence $\left\{u_{k}: k=1,2, \ldots.\right\} \subset G$ such that

$$
u_{0}=0, \quad u_{n} \notin \cup_{k=0}^{n-1}\left(K+u_{k}\right), \quad n=1,2, \ldots
$$

Let $x_{0}=0$, and set

$$
x_{n}=u_{n}-\tau\left(u_{n}\right), K_{n}=x_{n}+K_{0}, n=1,2, \ldots
$$

Then, it follows easily that

$$
\left\|f_{x_{n}}-f\right\|_{\infty}=\left\|f_{u_{n}}-f_{\tau\left(u_{n}\right)}\right\|_{\infty}<\varepsilon
$$

Also, note that

$$
K_{n} \cap K_{m}=\emptyset(n>m) .
$$

For, if not, $x_{n}+k_{1}=x_{m}+k_{2} \in K_{m} \cap K_{n}$ holds for some $k_{1}, k_{2} \in K_{0}$. From this relation with (20), we have $u_{n}=u_{m}-\tau\left(u_{m}\right)+\tau\left(u_{n}\right)+k_{2}-k_{1} \in u_{m}+K$, which contradicts (19).

Now, by (18) we have

$$
\left|f\left(x-x_{n}\right)\right| \geq\left|f\left(z_{0}\right)\right|-\varepsilon \quad\left(x \in K_{n}\right), n=1,2, \ldots
$$

By (21), $\left|f\left(x-x_{n}\right)-f(x)\right| \leq\left\|f_{x_{n}}-f\right\|_{\infty}<\varepsilon(x \in G)$. This relation with (23) gives

$$
|f(x)| \geq\left|f\left(x-x_{n}\right)\right|-\varepsilon \geq\left|f\left(z_{0}\right)\right|-2 \varepsilon \quad\left(x \in K_{n}\right), n=1,2, \ldots
$$

On the other hand, (17) implies

$$
|f(x)-g(x)| \leq\|f-g\|_{\infty}=\left|f\left(z_{0}\right)\right|-3 \varepsilon \quad(x \in G) .
$$

By (24) and (25), we have

$$
|g(x)| \geq|f(x)|-\left|f\left(z_{0}\right)\right|+3 \varepsilon \geq \varepsilon\left(x \in K_{n}\right), \quad n=1,2, \ldots
$$

From (26), we can lead a desired contradiction:

$$
\|g\|_{q}^{q} \geq \sum_{n=1}^{\infty} \int_{K_{n}} \varepsilon^{q} d x=\infty .
$$

Q.E.D. 
Lemma 8.2. (cf. [10] Theorem 4 ) Let $t$ be a function in $L^{\infty}(G)$. and let $T_{t}$ be a linear functional of $S^{p}(G)$ defined by

$$
T_{t}(f)=\int_{G} f(x) t(x) d x \quad\left(f \in S^{p}(G)\right)
$$

then we have

$$
\left\|T_{t}\right\|_{S^{p}(G)^{*}}=\inf \left\{\|g\|_{\infty}+\|h\|_{q}: t=g+h, g \in L^{\infty}(G), h \in L^{q}(G)\right\}
$$

where, $q$ is the conjugate exponent of $p$.

Proof. $\leq$. For each expression $t=g+h, g \in L^{\infty}(G), h \in L^{q}(G)$, we have

$$
\begin{aligned}
\left|T_{t} f\right| & \leq\left|\int_{G} f(x) g(x) d x\right|+\left|\int_{G} f(x) h(x) d x\right| \\
& \leq\|f\|_{1}\|g\|_{\infty}+\left\|f_{p}\right\| h \|_{q} \\
& \leq\|f\|_{S}\left(\|g\|_{\infty}+\|h\|_{q}\right)
\end{aligned}
$$

for every $f \in S^{p}(G)$. thus the inequality $\leq$ holds.

2. Let $A=L^{1}(G) \oplus L^{p}(G)$ be the Banach space with the norm defined by

$$
\left\|\left(f_{1}, f_{2}\right)\right\|=\max \left\{\left\|f_{1}\right\|,\left\|f_{2}\right\|\right\} \quad\left(\left(f_{1}, f_{2}\right) \in L^{1}(G) \oplus L^{p}(G)\right) .
$$

$S^{p}(G)$ can be embedded in $A$ by the isometric isomorphism

$$
S: S^{p}(G) \longmapsto A, \quad f \rightarrow(f, f) .
$$

Since the dual spaces of $L^{1}(G)$ and $L^{p}(G)$ are $L^{\infty}(G)$ and $L^{q}(G)$ respectively ([7] p. 148 (12.18) Theorem ), the dual space of $A$ can be identified naturally with the Banach space $L^{\infty}(G) \oplus L^{q}(G)$, in which the norm of elements are defined by :

$$
\|(g, h)\|=\|g\|_{\infty}+\|h\|_{q} \quad\left((g, h) \in L^{\infty}(G) \oplus L^{q}(G)\right) .
$$

Therefore the linear functional $T_{t} \circ S^{-1}$ of the closed subspace $\left\{(f, f) ; f \in S^{p}(G)\right\}$ has a Hahn-Banach extension to $A$, which can be expressed by an element of $L^{\infty}(G) \oplus L^{q}(G)$ say, $\left(g_{0}, h_{0}\right)$, and hence

$$
\begin{aligned}
T_{t}(f) & =\int_{G} f(x) g_{0}(x) d x+\int_{G} f(x) h_{0}(x) d x \\
& =\int_{G} f(x)\left(g_{0}(x)+h_{0}(x)\right) d x \quad\left(f \in S^{p}(G)\right)
\end{aligned}
$$


Since $S^{p}(G)$ contains every characteristic function of a Borel set of finite Haar measure, we have $t(x)=g_{0}(x)+h_{0}(x)$ a. e. on $G$. As the norm of $T_{t}$ is the same as its extension, we have

$$
\left\|T_{t}\right\|=\left\|g_{0}\right\|_{\infty}+\left\|h_{0}\right\|_{q}
$$

and inequality $\geq$ follows. Q.E.D.

Theorem 8.3. Let $G$ be a non-compact locally compact abelian group, and let $1<p<\infty$. For $S^{p}(G)$, we have the following relations:

(i) $C_{B S E}\left(\Phi_{S^{p}(G)}\right)=M(G)^{\wedge}=M\left(S^{p}(G)\right)^{\wedge}$.

(ii) $S^{p}(G)^{\wedge} C_{\neq} C_{B S E}^{0}\left(\Phi_{S^{p}(G)}\right)=L^{1}(G)^{\wedge}$.

Proof. (i) Since $\Phi_{S^{p}(G)}=\hat{G}$, we can identify $\operatorname{span}(\hat{G})$ with $\operatorname{span}\left(\Phi_{S^{p}(G)}\right)$. If $q$ is the conjugate exponent of $p$ and $t \in \operatorname{span}(\hat{G})$, we can caluculate $\|t\|_{S^{p}(G)^{*}}$ using Proposition 8.1 and Lemma 8.2 as follows:

$$
\left.\|t\|_{S^{p}(G)^{*}}=\inf \left\{\|f\|_{\infty}+\|g\|_{q}\right\}: t=f+g, f \in L^{\infty}(G), g \in L^{q}(G)\right\}=\|t\|_{\infty} .
$$

Therefore we have $C_{B S E}\left(\Phi_{S^{p}(G)^{*}}\right)=M(G)^{\wedge}$ by the BSE-theorem. But it is known that $M\left(S^{p}(G)\right)^{\wedge}=M(G)^{\wedge}$ (cf. [8] Corollary 3.5.1), and hence (i) holds.

(ii) In the same way as in (i) above, and with Corollary 6.7 we have

$$
S^{p}(G)^{\wedge} \subset_{\neq} L^{1}(G)^{\wedge}=C_{B S E}^{0}\left(\Phi_{S^{p}(G)}\right)
$$

and thus (ii) holds. Q.E.D.

Theorem 8.4. Let $A=L^{1}\left(N_{k}\right)$ be the semigroup algebra of $N_{k}$, where $k$ is a positive integer and $N_{k}=\{k, k+1, \ldots\}$ is an additive subsemi-group of $Z$. Then we have $\hat{A} \not \subset C_{B S E}^{0}\left(\Phi_{A}\right)$.

Proof. Let $k$ be a natural number. Set $\Delta_{0}=\{z \in C: 0<|z| \leq 1\}$ and for each $z \in \Delta_{0}$, put

$$
\varphi_{z}(a)=\sum_{n=k}^{\infty} a_{n} z^{n}\left(a=\left(a_{k}, a_{k+1}, \ldots\right) \in L^{1}\left(N_{k}\right)\right) .
$$

Then $\Phi_{L^{1}\left(N_{k}\right)}=\left\{\varphi_{z}: z \in \Delta_{0}\right\}$, and note that

$$
\|p\|_{L^{1}\left(N_{k}\right)^{*}}=\sup _{n \geq k}\left|\sum_{z \in \Delta_{0}} \hat{p}\left(\varphi_{z}\right) z^{n}\right|
$$


for every $p \in \operatorname{span}\left(\Phi_{L^{1}\left(N_{k}\right)}\right)$. Let $\delta_{k}=(1,0,0, \ldots)$. Then we assert that $\widehat{\delta_{k}}$ is not an element of $C_{B S E}^{0}\left(\Phi_{A}\right)$. Suppose the contrary. Then by Corollary 5.2 , there exists a $\mathcal{Q}_{c}$-neighbourhood $U_{K, \delta}$ such that

$$
\left|\sum_{z \in \Delta_{0}} \hat{p}\left(\varphi_{z}\right) z^{k}\right|<1 / 2
$$

for every $p \in U_{K, \delta}$. Take a small $\rho(1 / \sqrt{2}>\rho>0)$ such that $\left\{\varphi_{z}:|z| \leq \rho\right\} \subseteq K^{c}$. Moreover choose a natural number $n_{0}$ such that

$$
\frac{1}{2 \rho^{2 k}} \leq n_{0}<\frac{1}{\rho^{2 k}}
$$

and let $z_{0}, z_{1}, \ldots, z_{n_{0}-1}$ be $n_{0}$-points on the circle: $|z|=\rho$ and set

$$
c_{0}=\bar{z}_{0}^{k}, c_{1}=\bar{z}_{1}^{k}, \ldots, c_{n_{0}-1}=\bar{z}_{n_{0}-1}^{k} \text {. }
$$

We consider the following functional $p_{0} \in \operatorname{span}\left(\Phi_{A}\right)$ defined by

$$
p_{0}=\sum_{n=0}^{n_{0}-1} c_{n} \varphi_{z_{n}}
$$

In this case, $p_{0} \in U_{K, \delta}$ because $\widehat{p_{0}}=0$ on $K$ and

$$
\begin{aligned}
\left\|p_{0}\right\|_{L^{1}\left(N_{k}\right)^{*}} & =\sup _{n \geq k}\left|\sum_{z \in \Delta_{0}} \widehat{p_{0}}\left(\varphi_{z}\right) z^{n}\right| \leq \sum_{z \in \Delta_{0}}\left|\widehat{p_{0}}\left(\varphi_{z}\right) \| z\right|^{k} \\
& =\sum_{n=0}^{n_{0}-1}\left|c_{n} \| z_{n}\right|^{k} \leq n_{0} \rho^{2 k}<1
\end{aligned}
$$

Therefore we have

$$
\left|\sum_{z \in \Delta_{0}} \widehat{p_{0}}\left(\varphi_{z}\right) z^{k}\right|<1 / 2
$$

On the other hand, we have

$$
\left|\sum_{z \in \Delta_{0}} \widehat{p_{0}}\left(\varphi_{z}\right) z^{k}\right|=\left|\sum_{n=0}^{n_{0}-1} c_{n} z_{n}^{k}\right|=n_{0} \rho^{2 k} \geq 1 / 2 .
$$

This is a contradiction. Consequently, $\widehat{\delta_{k}} \in L^{1}\left(N_{k}\right)^{\wedge} \backslash C\left(\Phi_{L^{1}\left(N_{k}\right)} ; \mathcal{Q}_{c}\left(L^{1}\left(N_{k}\right)\right)\right)$. Q.E.D.

In the following, $f^{\prime}$ and $f^{\prime}(x)$ denotes the derivative of a function $f$ and the derivative of $f$ at $x$, respectively. 
Theorem 8.5. Let $A=C^{1}([0,1])$ be the set of functions consists of all the continuously differentialble functions on $[0,1]$. With pointwise addition, multiplication and the norm defined by $\|f\|=\max _{x \in[0,1]}\left(|f(x)|+\left|f^{\prime}(x)\right|\right) \quad(f \in A)$, A forms a unital Banach algebra (cf. [9] p.92) and the Gelfand space of $A$ can be identified with $[0,1]$. In this case, we have the following relations:

(i) $C_{B S E}\left(\Phi_{A}\right)=\operatorname{Lip}_{1}([0,1])=\{g \in C([0,1]): \rho(g)<\infty\}$, where $\rho(g)=\sup \{|(g(b)-g(a)) /(b-a)|: 0 \leq a<b \leq 1\}$.

(ii) $\hat{A}=\hat{M}(A) \subset_{\neq} C_{B S E}^{0}\left(\Phi_{A}\right)=C_{B S E}\left(\Phi_{A}\right)$.

Proof. (i) To simplify the expressions, we put $\mathcal{B}=\{g \in C([0,1]): \rho(g)<\infty\}$.

$\subseteq$. Let $\sigma \in C_{B S E}\left(\Phi_{A}\right)$ be arbitrary. For each $x_{1}, x_{2} \in[0,1]\left(x_{1}<x_{2}\right)$, we put $p=\frac{1}{x_{2}-x_{1}}\left(\delta_{x_{2}}-\delta_{x_{1}}\right)$, where $\delta_{x}(f)=f(x)(f \in A)$. Then

$$
\begin{aligned}
\|p\|_{A^{*}} & =\sup \left\{|p(f)|: f \in A,\|f\|_{A} \leq 1\right\} \\
& =\sup \left\{\left|\frac{f\left(x_{2}\right)-f\left(x_{1}\right)}{x_{2}-x_{1}}\right|: f \in A,\|f\|_{A} \leq 1\right\} \\
& \leq \sup \left\{\left|f^{\prime}\left(x_{1}+\theta\left(x_{2}-x_{1}\right)\right)\right|: 0<\theta<1, f \in A,\|f\|_{A} \leq 1\right\} \\
& \leq 1\left(\text { since }\left\|f^{\prime}\right\|_{\infty} \leq\|f\|_{A}\right),
\end{aligned}
$$

and hence

$$
\|\sigma\|_{B S E} \geq \sup \left\{\left|\sum_{x} \hat{p}(x) \sigma(x)\right|: p=\frac{\delta_{x_{2}}-\delta_{x_{1}}}{x_{2}-x_{1}}\left(0 \leq x_{1}<x_{2} \leq 1\right)\right\}=\rho(\sigma) .
$$

That is $\sigma \in \mathcal{B}$.

?. Let $\sigma \in \mathcal{B}$ be arbitrary, and let $\phi(x)$ be a function on $R$ defined by

$$
\phi(x)=\left\{\begin{array}{lc}
x+1, & \text { if }-1 \leq x<0 \\
-x+1, & \text { if } 0 \leq x \leq 1 \\
0, & \text { otherwise. }
\end{array}\right.
$$

Put $\phi_{n}(x)=n \phi(n x)(x \in R), n=1,2, \ldots$, and we have $\left\|\phi_{n}\right\|_{1}=1, n=1,2, \ldots$. We define the convolution of $\sigma$ with $\phi_{n}$ by

$$
\sigma * \phi_{n}(x)=\int_{0}^{1} \tilde{\sigma}(x-t) \phi_{n}(t) d t \quad(0 \leq x \leq 1),
$$

where $\tilde{\sigma}(x)$ is a function on $R$ such that $\tilde{\sigma}=\sigma$ on $[0,1]$ and $=0$ on $R \backslash[0,1]$.

Now we claim that the following (a), (b) and (c) hold:

(a) $\sigma * \phi_{n} \in C^{1}([0,1])=A$. 
(b) $\left\|\sigma * \phi_{n}\right\|_{A} \leq\|\sigma\|_{\infty}+\rho(\sigma)<\infty$

(c) $\lim _{n \rightarrow \infty} \sigma * \phi_{n}(x)=\sigma(x),(0 \leq x \leq 1)$

If we show that the above claims are true, we get by Theorem 4, (i) of [18] that $\sigma \in C_{B S E}\left(\Phi_{A}\right)$.

(a) Obviously, $\tilde{\sigma}$ is absolutely continuous, $\tilde{\sigma}^{\prime}=\sigma^{\prime}$ a.e. on $[0,1]$ and, $\tilde{\sigma}^{\prime}=0$ on $R \backslash[0,1]$. Moreover, it is easily to see that $\rho(\sigma) \geq\left|\sigma^{\prime}(x)\right|$ a.e. on $[0,1]$. Hence we get by Lebesgue's dominated convergence theorem:

$$
\begin{aligned}
\frac{d}{d x}\left(\sigma * \phi_{n}(x)\right) & =\lim _{h \rightarrow 0} \int_{0}^{1} \frac{\tilde{\sigma}(x+h-t)-\tilde{\sigma}(x-t)}{h} \phi_{n}(t) d t \\
& =\int_{0}^{1} \lim _{h \rightarrow 0} \frac{\tilde{\sigma}(x+h-t)-\tilde{\sigma}(x-t)}{h} \phi_{n}(t) d t \\
& =\int_{0}^{1} \tilde{\sigma}^{\prime}(x-t) \phi_{n}(t) d t \text { a.e. on }[0,1] .
\end{aligned}
$$

Therefore, the continuity of $\left(\sigma * \phi_{n}\right)^{\prime}(x)$ follows easily from (27), and we have $\sigma * \phi_{n} \in$ $C^{1}([0,1])=A$.

(b) $\left\|\sigma * \phi_{n}\right\|_{A} \leq\left\|\sigma * \phi_{n}\right\|_{\infty}+\left\|\left(\sigma * \phi_{n}\right)^{\prime}\right\|_{\infty}$

$$
\begin{aligned}
& \leq \sup _{x \in[0,1]}\left|\int_{0}^{1} \tilde{\sigma}(x-t) \phi_{n}(t) d t\right|+\sup _{x \in[0,1]}\left|\int_{0}^{1} \tilde{\sigma}^{\prime}(x-t) \phi_{n}(t) d t\right| \\
& \leq\|\sigma\|_{\infty}+\left\|\sigma^{\prime}\right\|_{\infty} \leq\|\sigma\|_{\infty}+\rho(\sigma)<\infty(n=1,2, \ldots) .
\end{aligned}
$$

(c) follows immediately by a direct calculation.

Thus the proof of (i) is complete.

(ii). Since $A$ has a unit, the relations $\hat{A}=\hat{M}(A)$ and $C_{B S E}^{0}\left(\Phi_{A}\right)=C_{B S E}\left(\Phi_{A}\right)$ are trivial, and the relation $\hat{A} \subset_{\neq} C_{B S E}^{0}\left(\Phi_{A}\right)$ follows from (i). Q.E.D.

Remark 8.1 Theorem 8.5 provides an example of a Banach algebra $A$ which satisfies all the conditions of Theorem 6.6 without being BSE. 


\section{References}

1. P. J. Cohen, On a conjecture of Littlewood and idempotent measures, Amer. J. Math., 82(1960), 191-212.

2. R. Doss, On the Fourier-Stieltjes transforms of singular or absolutely continuous measures, Math. Zeitschr., 97(1967),77-84.

3. R. Doss, On the transform of a singular or an absolutely continuous measure, Proc. Amer. Math.Soc., 19(1968), 361-363.

4. J. E. Gilbert, On a strong form of spectral synthesis, Ark. Mat. 7(1968), 571-575.

5. O. Hatori, Private communication.

6. S. Helgason, Multipliers of Banach algebras, Ann. Math., 64(1956), 240-254.

7. E. Hewitt and K. A. Ross, Abstract Harmonic Analysis, Springer- Verlag Berlin Heidelberg New York 1979.

8. R. Larsen, An Introduction to the Theory of Multipliers (Springer-Verlag, New York-Heiderberg, 1971.)

9. R. Larsen, Banach algebras, Marcel Dekker, Inc. New York 1973.

10. T.-S. Liu and J.-K. Wang, Sums and intersections of Lebegue spaces, Math. Scand. 23(1968), 241-251.

11. O. C. MacGhee, The restriction of a Fourier-Stieltjes transform to a measurable set, J. London Math. Soc.,(2), 12(1976), 253-256.

12. H. Reiter, $L^{1}$-Algebras and Segal Algebras, Lecture notes in Math. 231 (1971) Springer-Verlag Berlin ·Heidelberg.New York.

13. H. P. Rosenthal, A characterization of restrictions of Fourier-Stieltjes transforms, Pacific. J. Math., 22(1967) 403-418.

14. H. P. Rosenthal, On the existence of approximate identities in ideals of group algebras, Ark. Mat., 7(1967) 185-191.

15. W. Rudin, Functional Analysis, McGraw-Hill Inc. 1991. 
16. W. Rudin, Fourier Analysis on Groups, Interscience Publisher, Inc., New York, 1962.

17. S. Saeki, On strong Ditkin sets, Ark. Mat., 10-1(1972) 1-7.

18. S.-E. Takahasi and O. Hatori, Commutative Banach algebras which satisfy a Bochner-Schoenberg-Eberlein-type theorem, Proc. Amer. Math. Soc., 110 (1990), 149-158.

19. S.-E. Takahasi and O. Hatori, Commutative Banach algebras and BSE-inequalities, Math. Japonica, 37(1992), 47-52.

20. J.-K. Wang, Multipliers of commutative Banach algebras, Pacific J. Math., 1(1951), 305-311.

21. L. Y. H. Yap, Every Segal algebra satisfies Ditkin's condition, Studia Math. XL.(1971) 235-237. 